\title{
INOCULAÇÃO E ADUBAÇÃO MINERAL EM FEIJÃO-CAUPI: EFEITOS NA NODULAÇÃO, CRESCIMENTO E PRODUTIVIDADE ${ }^{1}$

\author{
INOCULATION AND MINERAL FERTILIZATION IN COWPEA: EFFECTS ON \\ NODULATION, PLANT GROWTH AND YIELD
}

\author{
Régia Maria Reis GUALTER ${ }^{2}$ \\ Luiz Fernando Carvalho LEITE ${ }^{3}$ \\ Ademir Sérgio Ferreira de ARAÚJO ${ }^{4}$ \\ Rosa Maria Cardoso Mota de ALCANTARA ${ }^{3}$ \\ Daniela Batista COSTA ${ }^{5}$
}

\begin{abstract}
RESUMO
A nodulação e o crescimento das leguminosas têm influência dos nutrientes contidos no solo, principalmente $\mathrm{P}, \mathrm{Ke}$ Mo. O presente trabalho objetivou avaliar em campo o efeito da inoculação com Bradyrhizobium elkanii e da adubação com $\mathrm{P}, \mathrm{K}$ e Mo sobre a nodulação, crescimento e produtividade de feijão-caupi (cultivar BRS Guariba). O estudo foi conduzido em uma área experimental, utilizando-se um Neossolo Flúvico, em Teresina, PI. Os tratamentos foram dispostos em um delineamento em blocos casualizados com seis tratamentos e quatro repetições. Os tratamentos consistiram de aplicação ou não de $P, K$ e Mo no solo e de inoculante rizobiano nas sementes. No tratamento com inoculação foi utilizado um inoculante a base de turfa contendo Bradyrhizobium elkanii, estirpe BR 3262. A semeadura foi realizada em parcela experimental de 3,2 $\mathrm{m} \times 5,0 \mathrm{~m}$ e procederam-se as coletas dos dados aos 35 e 50 dias após a emergência (DAE) para a determinação do número e massa seca dos nódulos e das massas secas da parte aérea e raiz e acúmulo de $\mathrm{N}$ na parte aérea. A produtividade de grãos foi determinada aos 58 DAE. Não se verificou diferença entre os tratamentos para massa seca das raízes, parte aérea e acúmulo de $\mathrm{N}$ aos $35 \mathrm{DAE}$, para massa seca das raízes aos $50 \mathrm{DAE}$ e para a produtividade de grãos. Em solo com adequado conteúdo de $\mathrm{P}$ e $\mathrm{K}$ a inoculação com Bradyrhizobium elkanii associada à adubação fosfatada, potássica e molíbidica não contribuiu para o aumento do número e massa dos nódulos e nem tampouco da produtividade de grãos de feijão-caupi.
\end{abstract}

Palavras-chave: Bradyrhizobium elkanii; leguminosas; Vigna unguiculata.

\begin{abstract}
The nodulation and growth of legumes have influences of nutrients contained in the soil, mainly $\mathrm{P}, \mathrm{K}$ and Mo. The present study aimed to evaluate, in field, the effect of inoculation with Bradyrhizobium elkanii and soil fertilized with $\mathrm{P}, \mathrm{K}$ and Mo on nodulation, plant growth and yield in cowpea (BRS Guariba). The experiment was carried out in an experimental area at Teresina, $\mathrm{PI}$. The treatments were disposed in a completely randomized block with six treatments and four repetitions. The treatments consisted of presence and absence of soil fertilization with $\mathrm{P}, \mathrm{K}$ and Mo and planted with seeds inoculated or noninoculated. In the treatment with inoculation, an inoculant was used containing Bradyrhizobium elkanii, strain BR 3262. The sowing was conduced out in plots of $3.2 \mathrm{~m} \times 5.0 \mathrm{~m}$ and the collect of data was conduced to the 35 and 50 days after plant emergence (DAE) for the determination of nodulation and plant growth. The yield was determined $58 \mathrm{DAE}$. Differences were not verified among the treatments to root and shoot dry weight and increase in N at $35 \mathrm{DAE}$, to root dry weight at $50 \mathrm{DAE}$ and to grain yield. In soil with appropriate $\mathrm{P}, \mathrm{K}$ content, the inoculation with Bradyrhizobium elkanii plus $\mathrm{P}, \mathrm{K}$ and Mo fertilization not contributed to increase in the nodule number and dry weight and nor either of the grain yield of cowpea.
\end{abstract}

Key-words: Bradyrhizobium elkanii; legumes; Vigna unguiculata.

\footnotetext{
${ }^{1}$ Trabalho de Iniciação Científica com bolsa ITI/CNPq.

Estudante de Biologia, Universidade Federal do Piauí, Bolsista ITI/CNPq.

${ }^{3}$ Engenheiro Agrônomo, Doutor, Pesquisador da Embrapa Meio-Norte. Av. Duque de Caxias, Caixa postal 001, 64006-220, Teresina-PI. E-mail: luizf@cpamn.embrapa.br, rosa@cpamn.embrapa.br

${ }^{4}$ Engenheiro Agrônomo, Doutor, Professor da Universidade Federal do Piauí. Centro de Ciências Agrárias, Campus da Socopo, Teresina - PI. CEP: 64.049-550. e-mail: asfaruaj@yahoo.com.br (autor para correspondência).

${ }^{5}$ Engenheiro Agrônomo, Mestre, Bolsista DTI - M/ CNPq, Embrapa Meio-Norte.Caixa postal 001, 64006-220, Teresina-PI.
} 


\section{INTRODUÇÃO}

A simbiose rizóbio-leguminosas é um exemplo de associação biológica cujos benefícios para a sustentabilidade agrícola são reconhecidos devido ao processo de fixação biológica do nitrogênio (FBN) sendo possível substituir parcial ou totalmente a adubação nitrogenada (HUNGRIA et al., 1999).

O feijão-caupi, dentre as leguminosas que se beneficiam do processo, tem grande importância socioeconômica e potencial estratégico, principalmente para as regiões Norte e Nordeste, onde constitui um dos mais importantes componentes da dieta alimentar (FREIRE FILHO et al., 2005). Nestas regiões, a cultura apresenta baixa produtividade média sendo uma das causas a baixa disponibilidade de nutrientes no solo, principalmente o N. O aumento da eficiência do processo de nodulação e conseqüentemente da FBN é uma das formas de aumentar a produtividade da cultura (FRANCO et al., 2002). Além disso, melhorando-se o desempenho simbiótico pode-se, da mesma forma que ocorre com a soja, dispensar a necessidade do uso de adubos nitrogenados para obtenção de maiores rendimentos (RUMJANEK et al., 2005).

Entretanto, a nodulação e a FBN são influenciadas por fatores edafoclimáticos que podem trazer benefícios ou prejuízos ao processo. A disponibilidade de nutrientes está entre os principais fatores edáficos que influenciam a FBN com destaque para o $\mathrm{P}$, o $\mathrm{K}$ e o Mo. O $\mathrm{P}$ auxilia na nodulação pela transferência de energia na forma de ATP e no aumento no número de pêlos radiculares proporcionando mais sítios de infecção para a bactéria (OTHMAN et al., 1991; OKELEYE e OKELANA, 1997) e, quando presente em níveis insuficientes, é capaz de comprometer a eficiência da associação simbiótica (FREIRE FILHO et al., 2005). A deficiência de $K$ afeta a fotossíntese e, conseqüentemente, o fornecimento de fotossintatos da planta para a bactéria, limitando a nodulação e a fixação simbiótica do nitrogênio (DUKE e COLLINS, 1985).

O Mo é considerado fundamental, pois é um elemento-chave do centro ativo da nitrogenase, enzima responsável pela fixação biológica de nitrogênio (MENGEL e KIRKBY, 2001). Além disso, o elemento atua na redutase do nitrato, uma enzima responsável pela redução do $\mathrm{NO}_{3}$ para ser assimilado pela planta (DECHEN et al., 1991). Desta forma, no caso de leguminosas, o Mo é necessário para a manutenção da atividade de duas enzimas relacionadas ao aproveitamento do $\mathrm{N}$ podendo, portanto, sua aplicação promover maior acúmulo de $\mathrm{N}$ nas plantas. A influência positiva do Mo no aumento da quantidade de $\mathrm{N}$ na parte aérea de plantas de uma variedade de feijão foi verificada por KUSDRA (2003). Por interferir favoravelmente no metabolismo do nitrogênio das plantas, especialmente em leguminosas, o Mo pode também ser capaz de aumentar a produtividade de grãos do feijão-caupi.

O objetivo deste estudo foi avaliar o efeito da inoculação e da adubação mineral sobre a nodulação, crescimento e produtividade do feijãocaupi.

\section{MATERIAL E MÉTODOS}

O experimento foi instalado na Embrapa Meio-Norte, localizada em Teresina, PI (0505'S e $42^{\circ} 48^{\prime} \mathrm{W}$ e altitude de $74,4 \mathrm{~m}$ ) de setembro a dezembro de 2006. O clima caracteriza-se por apresentar temperatura média de $27,9^{\circ} \mathrm{C}$, umidade relativa do ar média de $69,2 \%$ e precipitação média anual de $1300 \mathrm{~mm}$ (BASTOS e ANDRADE JÚNIOR, 2000). O solo da área é classificado como Neossolo Flúvico eutrófico (EMBRAPA, 1999) e apresentava as seguintes características químicas (camada 0-20 $\mathrm{cm})$ : M.O: $15,51 \mathrm{~g} \mathrm{~kg}^{-1}$; $\mathrm{pH}: 5,45 ; \mathrm{P}: 37,38 \mathrm{mg} \mathrm{dm}^{-3} ; \mathrm{K}$ : $0,5 \mathrm{cmol}_{\mathrm{c}} \mathrm{dm}^{-3} ; \mathrm{Ca}: 2,36 \mathrm{cmol}_{\mathrm{cm}} \mathrm{dm}^{-3} \mathrm{Mg}: 1,71 \mathrm{cmol}_{\mathrm{c}}$ $\mathrm{dm}^{-3}$; $\mathrm{Na}: 0,17 \mathrm{cmol}^{-3}$; $\mathrm{Al}: 0,06 \mathrm{cmol}^{\mathrm{c}} \mathrm{dm}^{-3} ; \mathrm{H}+\mathrm{Al}$ : $2,43 \mathrm{cmol}^{-3 m^{-3}}$ e CTC: $7,17 \mathrm{cmol}^{\mathrm{c}} \mathrm{dm}^{-3}$.

O preparo do solo foi constituído por uma aração e duas gradagens leves, sendo a primeira logo após a aração, e a segunda um dia antes da semeadura para a incorporação do herbicida Roundup.

Os tratamentos avaliados estão apresentados na Tabela 1. As doses de P, K e Mo de 20,40 e $10 \mathrm{~kg} \mathrm{ha}^{-1}$, respectivamente, aplicadas na semeadura, foram definidas de acordo com FREIRE FILHO et al. (2005) e com base na análise de solo, realizada antes da implantação do experimento. As fontes de $\mathrm{P}, \mathrm{K}$ e Mo foram o superfosfato simples $\left(\mathrm{P}_{2} \mathrm{O}_{5}\right)$, cloreto de potássio $\left(\mathrm{K}_{2} \mathrm{O}\right)$ e FTE BR-12, respectivamente. As sementes de feijão-caupi (BRS Guariba) foram inoculadas com inoculante turfoso (concentração de $10^{9}$ células $\mathrm{g}^{-1}$, conforme 0 fabricante), o qual continha a estirpe BR 3262 de Bradyrhizobium elkanii. A dosagem utilizada foi de $500 \mathrm{~g}$ de inoculante para $50 \mathrm{~kg}$ de sementes e a inoculação foi realizada com o umedecimento prévio das sementes com uma solução açucarada (10\% $\mathrm{p} \mathrm{v}^{-1}$ ) na proporção de $6 \mathrm{~mL} \mathrm{~kg}^{-1}$ de semente (HUNGRIA et al., 2001).

A parcela experimental correspondeu a 15 $\mathrm{m}^{2}$, com quatro fileiras de $5,0 \mathrm{~m}$ de comprimento, tendo como área útil as duas fileiras centrais. A semeadura ocorreu logo após a inoculação das sementes, colocando-se cerca de quatro sementes por cova e semeada no espaçamento de $0,75 \mathrm{~m} \mathrm{x}$ $0,5 \mathrm{~m}$ sendo conduzida sob irrigação por aspersão.

Durante o desenvolvimento da cultura foram realizadas capinas com auxílio de enxadas, procurando-se manter a cultura livre de plantas invasoras e tratos fitossanitários, visando ao controle de pragas (pulgões, cigarrinhas e vaquinhas) que ocorrem na cultura do feijão-caupi na região. 
TABELA 1 - Descrição dos tratamentos. Teresina, 2006.

\begin{tabular}{ll}
\hline Tratamentos & Descrição \\
\hline T1 & Não inoculado - sem PK - sem Mo (Testemunha) \\
T2 & Não inoculado - sem PK - com Mo \\
T3 & Não inoculado - com PK - com Mo \\
T4 & Inoculado com Bradyrhizobium elkanii estirpe BR-3262 - sem PK - sem Mo \\
T5 & Inoculado com Bradyrhizobium elkanii estirpe BR-3262 - sem PK - com Mo \\
T6 & Inoculado com Bradyrhizobium elkanii estirpe BR-3262 - com PK - com Mo \\
\hline
\end{tabular}

Foram coletadas três plantas de cada parcela aos 35 e 50 DAE (dias após a emergência), correspondentes aos estádios R1 e R3 (XAVIER et al., 2007) para a cultivar BRS-Guariba. As variáveis avaliadas foram: número de nódulos (NN), massa seca de nódulos (MSN), massa seca das raízes (MSR), massa seca da parte aérea (MSPA) e acúmulo de $\mathrm{N}$ na parte aérea (ANPA). A produtividade de grãos foi avaliada aos $58 \mathrm{DAE}$, com umidade corrigida para $13 \%$.

$\mathrm{O} N$ total foi determinado pelo método semimicrokjeldahl (SILVA, 1981) e utilizado para se calcular o ANPA. O N acumulado na matéria seca da parte aérea foi calculado, multiplicando-se sua massa pelo teor de $\mathrm{N}$.

A parte aérea foi separada das raízes em corte no ponto de inserção cotiledonar, próximo à base do caule. Os nódulos foram retirados das raízes, contados, secos em papel absorvente e pesados. Para determinação da produção de matéria seca, o material vegetal foi colocado em estufa com circulação forçada de ar à 65-70 ${ }^{\circ} \mathrm{C}$ até atingir massa constante.

O delineamento experimental foi o de blocos completos casualizados com 6 tratamentos e quatro repetições. Os dados foram submetidos à análise de variância empregando-se o programa de análise estatística ASSISTAT, versão 7.4 beta (SILVA, 2007) e as médias foram comparadas pelo teste de Tukey a $5 \%$.

\section{RESULTADOS E DISCUSSÃO}

Na primeira avaliação, aos 35 DAE, não se verificou diferenças significativas no número e massa dos nódulos entre os tratamentos com e sem inoculação (Tabela 2). Este resultado mostra que, nos tratamentos não inoculados, a presença de bactérias nativas no solo proporcionou a nodulação nas raízes do feijão-caupi. Resultados semelhantes foram observados por XAVIER et al. (2006) avaliando a nodulação por estirpes de Bradyrhizobium spp. em feijão-caupi e comparando com as bactérias nativas do solo. Os autores citam que, embora a concentração das bactérias no inoculante seja de
$10^{9}$ células $\mathrm{mL}^{-1}$, as estirpes podem não apresentar diferenças em relação às bactérias nativas na nodulação, mesmo estas estando em baixa população no solo. Segundo RUMJANEK et al. (2005), não só a densidade de células no inoculante é responsável por promover nodulação satisfatória, mas outros eventos como, por exemplo, a interferência dos fatores ambientais e do solo e a capacidade de sobrevivência da estirpe.

De modo geral, na segunda avaliação aos 50 DAE, houve uma diminuição no número e na massa dos nódulos em todos os tratamentos, em comparação com a primeira avaliação aos $35 \mathrm{DAE}$ (Tabela 2), sugerindo o início da senescência nodular. Esta situação deveu-se, provavelmente, ao ciclo curto da cultivar avaliada (aproximadamente 60 dias). Geralmente, após o florescimento e o início do enchimento de grãos ocorre o período de senescência nodular, uma vez que os fotossintatos acumulados pela planta hospedeira são direcionados para os grãos, desfavorecendo os nódulos (HUNGRIA e VARGAS, 1994).

Não houve diferenças significativas entre os tratamentos, nas duas avaliações, para a massa seca das raízes. Já a produção de biomassa seca da parte aérea mostrou diferenças, entre os tratamentos, aos 50 DAE (Tabela 3). Entretanto, para a biomassa da parte aérea os resultados foram variáveis, embora tenha havido um incremento na massa seca da parte aérea no tratamento com inoculação de estirpe 3262 e sem adição de $P, \mathrm{~K}$ e Mo. Por um lado, os resultados indicam que os nutrientes avaliados neste estudo não influenciaram no acúmulo de biomassa vegetal do feijão-caupi. Este fato pode ser devido ao adequado conteúdo de $\mathrm{P}$ e K apresentado pelo solo avaliado, pois, segundo FREIRE FILHO et al. (2005), nível igual ou superior a 10 e $20 \mathrm{mg} \mathrm{dm}^{-3}$ para $P$ e $\mathrm{K}$, respectivamente, não proporciona resposta das plantas a aplicação dos nutrientes. Entretanto, o nutriente mais diretamente relacionado ao aumento da biomassa vegetal é o $\mathrm{N}$ (EPSTEIN e BLOOM, 2006) e, desta forma, a presença de nódulos em número e massa satisfatória em todos os tratamentos (Tabela 2) pode ter proporcionado FBN eficiente e disponibilizado $\mathrm{N}$ 
GUALTER, R.M.R. et al. Inoculação e adubação mineral e feijão-caupi...

TABELA 2 - Número de nódulos (NN), massa seca de nódulos (MSN), aos 35 e 50 dias após a emergência (DAE) em feijão-caupi. Teresina-PI, 2006.

\begin{tabular}{|c|c|c|}
\hline Tratamentos & $\begin{array}{c}\mathrm{NN} \\
\left(\mathrm{n}^{0} \text { planta }^{-1}\right)\end{array}$ & $\begin{array}{c}\text { MSN } \\
\left(\text { mg planta }^{-1}\right)\end{array}$ \\
\hline & \multicolumn{2}{|c|}{$35 \mathrm{DAE}$} \\
\hline T1: não inoculado - sem PK - sem Mo & $23 a b$ & $71,00 a b$ \\
\hline T2: não inoculado - sem PK - com Mo & $17 \mathrm{~b}$ & $89,00 \mathrm{ab}$ \\
\hline T3: não inoculado - com PK - com Mo & $18 \mathrm{~b}$ & $50,25 a b$ \\
\hline T4: inoculado - sem PK - sem Mo & $37 a b$ & $46,25 a b$ \\
\hline T5: inoculado - sem PK - com Mo & $39 a b$ & $36,00 \mathrm{~b}$ \\
\hline T6: inoculado - com PK - com Mo & $45 \mathrm{a}$ & $106,00 \mathrm{a}$ \\
\hline \multirow[t]{2}{*}{$\mathrm{CV}(\%)$} & 38,2 & 42,0 \\
\hline & \multicolumn{2}{|c|}{$50 \mathrm{DAE}$} \\
\hline T1: não inoculado - sem PK - sem Mo & $20 a b$ & $40,32 \mathrm{ab}$ \\
\hline T2: não inoculado - sem PK - com Mo & $7 \mathrm{c}$ & $19,00 \mathrm{bc}$ \\
\hline T3: não inoculado - com PK - com Mo & $28 \mathrm{a}$ & $51,32 \mathrm{a}$ \\
\hline T4: inoculado - sem PK - sem Mo & $20 a b$ & $39,65 \mathrm{abc}$ \\
\hline T5: inoculado - sem PK - com Mo & $11 \mathrm{bc}$ & $17,00 \mathrm{bc}$ \\
\hline T6: inoculado - com PK - com Mo & $9 \mathrm{c}$ & $12,65 \mathrm{c}$ \\
\hline $\mathrm{CV}(\%)$ & 24,2 & 39,0 \\
\hline
\end{tabular}

Médias seguidas pela mesma letra, na coluna, não diferem entre si pelo teste de Tukey a $5 \%$ de probabilidade.

TABELA 3 - Massa seca das raízes (MSR) e da parte aérea (MSPA) e acúmulo de N (ANPA) aos 35 e 50 dias após a emergência (DAE) em feijão-caupi. Teresina-PI, 2006.

\begin{tabular}{lccc}
\hline Tratamentos & $\begin{array}{c}\text { MSR } \\
\left(\mathrm{g} \mathrm{planta}^{-1}\right)\end{array}$ & $\begin{array}{c}\text { MSPA } \\
\left(\mathrm{g} \mathrm{planta}^{-1}\right)\end{array}$ & $\begin{array}{c}\text { ANPA } \\
(\%)\end{array}$ \\
\cline { 2 - 4 } T1: não inoculado - sem PK - sem Mo & $6,40 \mathrm{a}$ & $86,87 \mathrm{a}$ & $2,95 \mathrm{a}$ \\
T2: não inoculado - sem PK - com Mo & $8,65 \mathrm{a}$ & $118,07 \mathrm{a}$ & $3,87 \mathrm{a}$ \\
T3: não inoculado - com PK - com Mo & $6,97 \mathrm{a}$ & $133,27 \mathrm{a}$ & $4,05 \mathrm{a}$ \\
T4: inoculado - sem PK - sem Mo & $7,97 \mathrm{a}$ & $134,25 \mathrm{a}$ & $4,15 \mathrm{a}$ \\
T5: inoculado - sem PK - com Mo & $6,30 \mathrm{a}$ & $9,00 \mathrm{a}$ \\
T6: inoculado - com PK - com Mo & $6,60 \mathrm{a}$ & $111,62 \mathrm{a}$ & $3,90 \mathrm{a}$ \\
\hline CV(\%) & 23,2 & 27,4 & 27,0 \\
\hline T1: não inoculado - sem PK - sem Mo & & $50 \mathrm{DAE}$ & $3,55 \mathrm{ab}$ \\
T2: não inoculado - sem PK - com Mo & $9,05 \mathrm{a}$ & $158,05 \mathrm{ab}$ & $2,30 \mathrm{~b}$ \\
T3: não inoculado - com PK - com Mo & $10,12 \mathrm{a}$ & $124,27 \mathrm{~b}$ & $2,97 \mathrm{ab}$ \\
T4: inoculado - sem PK - sem Mo & $8,87 \mathrm{a}$ & $160,50 \mathrm{ab}$ & $4,47 \mathrm{a}$ \\
T5: inoculado - sem PK - com Mo & $9,85 \mathrm{a}$ & $200,75 \mathrm{a}$ & $2,45 \mathrm{~b}$ \\
T6: inoculado - com PK - com Mo & $7,97 \mathrm{a}$ & $95,85 \mathrm{~b}$ & $3,02 \mathrm{ab}$ \\
\hline CV(\%) & $11,40 \mathrm{a}$ & $140,62 \mathrm{ab}$ & 21,3
\end{tabular}

Médias seguidas pela mesma letra, na coluna, não diferem entre si pelo teste de Tukey a $5 \%$ de probabilidade. 
para as plantas, conforme observado por SOUZA et al. (2008) para soja e XAVIER et al. (2007) para feijãocaupi. Resultados semelhantes para o número e massa dos nódulos foram encontrados por XAVIER et al. (2008) que avaliaram a nodulação e a produtividade do feijão-caupi em um Neossolo quartzarênico localizado na mesma região de realização deste trabalho.

Comportamento semelhante ao da biomassa da parte aérea foi observado para o acúmulo de $\mathrm{N}$ na parte aérea (Tabela 3 ) sugerindo que o fornecimento de $\mathrm{N}$ para as plantas se deu, provavelmente, via FBN, uma vez que houve uma nodulação satisfatória em todos os tratamentos. Além disso, não foi realizada adubação nitrogenada no plantio e a contribuição estimada do $\mathrm{N}$ proveniente da mineralização da matéria orgânica é insignificante devido o curto período do experimento. Segundo DOBEREINER (1966), o aumento da quantidade de $\mathrm{N}$ acumulado em leguminosas está diretamente relacionado com a nodulação, constatação esta confirmada por WADISIRISUK e WEAVER (1985) em feijão-caupi.

Não houve respostas das plantas para a nodulação, crescimento vegetal e produtividade devido à aplicação de Mo, apesar deste micronutriente contribuir para a FBN. Provavelmente, isto ocorreu devido ao solo apresentar quantidade suficiente de Mo para atender as necessidades das plantas em relação a este elemento.

A produtividade de grãos variou de 1.105 a $1.243 \mathrm{~kg} \mathrm{ha}^{-1}$, mas sem diferença entre os tratamentos (Tabela 4). Esta situação foi conseqüência, provavelmente, dos resultados observados na nodulação, biomassa vegetal e acúmulo de $\mathrm{N}$. Embora os níveis de produtividade alcançados neste trabalho sejam superiores a média da região $\left(300 \mathrm{~kg} \mathrm{ha}^{-1}\right)$ estes não podem ser atribuídos à estirpe BR 3262 de Bradyrhizobium elkanii presente no inoculante, uma vez que a nodulação de plantas sem a inoculação caracterizou a existência no solo de bactérias nativas capazes de promover nodulação no feijão-caupi. Entretanto, XAVIER et al. (2008), em Neossolo Quartzarênico de baixa fertilidade, avaliaram a produtividade do feijão-caupi com e sem inoculação e observaram maior produtividade (aproximadamente $1.400 \mathrm{~kg} \mathrm{ha}^{-1}$ ) no tratamento inoculado. Desta forma, os resultados encontrados neste experimento evidenciam a necessidade de estudos para isolamento de estirpes locais mais competitivas e eficientes na FBN para a região Meio Norte do Brasil.

TABELA 4 - Produtividade de grãos de feijão-caupi. Teresina-PI, 2006.

\begin{tabular}{lc}
\hline \multicolumn{1}{c}{ Tratamentos } & $\begin{array}{c}\text { Produtividade de grãos } \\
\mathrm{kg} \mathrm{ha}^{-1}\end{array}$ \\
\hline T1: não inoculado - sem PK - sem Mo & $1105,00 \mathrm{a}$ \\
T2: não inoculado - sem PK - com Mo & $1243,50 \mathrm{a}$ \\
T3: não inoculado - com PK - com Mo & $1205,00 \mathrm{a}$ \\
T4: inoculado - sem PK - sem Mo & $1231,75 \mathrm{a}$ \\
T5: inoculado - sem PK - com Mo & $1183,25 \mathrm{a}$ \\
T6: inoculado - com PK - com Mo & $1113,50 \mathrm{a}$ \\
\hline
\end{tabular}

$\begin{array}{ll}\mathrm{CV}(\%) & 17,6\end{array}$

Médias seguidas pela mesma letra, na coluna, não diferem entre si pelo teste de Tukey a $5 \%$ de probabilidade.

\section{CONCLUSÕES}

1- Em solo com adequado conteúdo de P e $\mathrm{K}$ a inoculação com Bradyrhizobium elkanii associada à adubação fosfatada, potássica e molíbidica não contribuiu para o aumento no número e na massa dos nódulos.

2- A estirpe BR 3262 de Bradyrhizobium elkanii e a adubação fosfatada, potássica e molíbidica não interferiram na produtividade da cultura de feijão-caupi, cultivar BRS-Guariba.

\section{AGRADECIMENTOS}

Ao Banco do Nordeste do Brasil (BNB) pelo financiamento do projeto e ao CNPq pela concessão da bolsa de Iniciação Tecnológica Industrial (ITI). 


\section{REFERÊNCIAS}

1. BASTOS, E. A.; ANDRADE JÚNIOR, A. S. Dados agrometeorológicos para o município de Teresina, PI (19801999). Teresina: Embrapa Meio-Norte, 2000. 27 p.

2. DECHEN, A. R.; HAAG, H. P.; CARMELO, Q. A. C. Mecanismos de absorção e de translocação de micronutrientes. In: FERREIRA, M. E. Micronutrientes na agricultura. Piracicaba: Potafos, 1991. p. 79-111.

3. DOBEREINER, J. Evaluation of nitrogen fixation in legumes by the regression of total plant nitrogen with nodule weight. Nature, v. 210, p. 850-852, 1966.

4. DUKE, S. H.; COLLINS, M. Role of potassium in legume dinitrogen fixation. In: MUNSON, R. D. (Ed.) Potassium in agriculture. Madison: American Society of Agronomy, 1985. p. 443-465.

5. EMBRAPA. Centro Nacional de Pesquisa de Solos. Sistema brasileiro de classificação de solos. Brasília: Embrapa Produção de Informação; Rio de Janeiro: Embrapa Solos, 1999. 412 p.

6. EPSTEIN, E.; BLOOM, A. J. Nutrição e crescimento. In: EPSTEIN, E.; BLOOM, A. J. (Ed.) Nutrição mineral de plantas. Londrina: Planta, 2006. p. 251-286.

7. FRANCO, M. C.; CASSINI, S. T. A.; OLIVEIRA, V. R.; VIEIRA, C.; TSAI, S. M. Nodulação em cultivares de feijão dos conjuntos gênicos andino e meso-americano. Pesquisa Agropecuária Brasileira, v. 37, n.8, p. 1145-1150, 2002.

8. FREIRE FILHO, F. R.; LIMA, J. A. A.; RIBEIRO, V. Q. Feijão-caupi: avanços tecnológicos. Brasília-DF: Embrapa Informação Tecnológica, 2005. $519 \mathrm{p}$

9. HUNGRIA, M.; CAMPO, R.J.; MENDES, I.C. Fixação biológica do nitrogênio na cultura da soja. Londrina: Embrapa Soja, 2001. 48 p.

10. HUNGRIA, M.; VARGAS, M. A. T. Fixação biológica do nitrogênio em soja. In: ARAUJO, R. S.; HUNGRIA, M. (Ed.). Microrganismos de importância agrícola. Brasília: Embrapa-SPI, 1994. p. 9-90.

11. HUNGRIA, M.; VARGAS, M. A. T.;ANDRADE, D. S.; CAMPO, R. J.; CHUEIRE, L. M. O.; FERREIRA, M. C.; MENDES, I. C. Fixação biológica do nitrogênio em leguminosas de grãos. In: SIQUEIRA, J. O.; MOREIRA, F. M. S.; LOPES, A. S.; GUILHERME, L. R. G.; FAQUIN, V.; FURTINI NETO, A. E.; CARVALHO, J. G. (Ed.) Inter-relação fertilidade, biologia do solo e nutrição de plantas. Lavras: SBCS, 1999. p. 597-620.

12. KUSDRA, J. F. Nodulação do feijoeiro e fixação biológica do nitrogênio em resposta à microbiolização das sementes e à aplicação de micronutrientes. Scientia Agraria, v. 4, p. 96, 2003.

13. MENGEL, K.; KIRKBY, E. A. Principles of plant nutrition. 5. ed. Dordrecht: Kluwer Academic Publishers, 2001. $849 \mathrm{p}$.

14. OKELEYE, K. A.; OKELANA, M. A. Effect of phosphorus fertilizer on nodulation, growth and yield of cowpea (Vigna unguiculata) varieties. Indian Journal of Agricultural Sciences, v. 67, p. 10-12, 1997.

15. OTHMAN, W. M. W.; LIE, T. A.; MANNETJE, L.; WASSINK, G. Y.; WAN-OTHMAN, W. M. Low level phosphorus supply affecting nodulation, $\mathrm{N}_{2}$ fixation and growth cowpea (Vigna unguiculata). Plant and Soil, v. 15, p. 67-74, 1991.

16. RUMJANEK, N. G.; MARTINS, L. M. V.; XAVIER, G. R.; NEVES, M. C. P. Fixação biológica do nitrogênio. In: FREIRE FILHO, F. R.; LIMA, J. A. A.; RIBEIRO, V. Q. (Ed.) Feijão-caupi: avanços tecnológicos. Brasília: Embrapa, 2005. p. 281-335.

17. SILVA, D. J. Análise de alimentos, métodos químicos e biológicos. Viçosa-MG: Editora da Universidade Federal de Viçosa-UFV, 1981. $166 \mathrm{p}$.

18. SILVA, F. de A. S. ASSISTAT Versão 7.4 beta. [Online]. 2007. Disponível em: <http://assistat.sites.uol.com.br>. Acesso em: 20/03/2007.

19. SOUZA, R. A.; HUNGRIA, M.; FRANCHINI, J. C.; MACIEL, C. D.; CAMPO, R. J.; ZAIA, D. A. M. Conjunto mínimo de parâmetros para avaliação da microbiota do solo e da fixação biológica do nitrogênio pela soja. Pesquisa Agropecuária Brasileira, v. 43, n. 1, p. 83-91, 2008.

20. XAVIER, G. R.; MARTINS, L. M. V.; RIBEIRO, J. R. A.; RUMJANEK, N. G. Especificidade simbiótica entre rizóbios e acessos de feijão-caupi de diferentes nacionalidades. Caatinga, v. 19, n. 1, p. 25-33, 2006.

21. XAVIER, T. F.; ARAÚJO, A. S. F.; SANTOS, V. B.; CAMPOS, F. L. Ontogenia da nodulação em duas cultivares de feiião-caupi. Ciência Rural, v. 37, n. 2, p. 561-564, 2007.

22. XAVIER, T. F.; ARAÚJO, A. S. F.; SANTOS, V. B.; CAMPOS, F. L. Influência da inoculação e adubação nitrogenada sobre a nodulação e produtividade de grãos de feijão-caupi. Ciência Rural, v. 38, 2008 (No prelo).

23. WADISIRISUK, P.; WEAVER, R. W. Importance of bacteroid number in nodules and effective nodule mass to dinitrogen fixation by cowpeas. Plant and Soil, v. 87, p. 223-231, 1985. 
